\title{
Secure Top-k Subgroup Discovery
}

\author{
Henrik Grosskreutz, Benedikt Lemmen and Stefan Rüping \\ Fraunhofer IAIS, Schloss Birlinghoven, Sankt Augustin, Germany \\ \{firstname.lastname\}@iais.fraunhofer.de
}

\begin{abstract}
Supervised descriptive rule discovery techniques like subgroup discovery are quite popular in applications like fraud detection or clinical studies. Compared with other descriptive techniques, like classical support/confidence association rules, subgroup discovery has the advantage that it comes up with only the top-k patterns, and that it makes use of a quality function that avoids patterns uncorrelated with the target. If these techniques are to be applied in privacy-sensitive scenarios involving distributed data, precise guarantees are needed regarding the amount of information leaked during the execution of the data mining. Unfortunately, the adaptation of secure multi-party protocols for classical support/confidence association rule mining to the task of subgroup discovery is impossible for fundamental reasons. The source is the different quality function and the restriction to a fixed number of patterns - i.e. exactly the desired features of subgroup discovery. In this paper, we present a new protocol which allows distributed subgroup discovery while avoiding the disclosure of the individual databases. We analyze the properties of the protocol, describe a prototypical implementation and present experiments that demonstrate the feasibility of the approach.
\end{abstract}

\section{Introduction}

The question of the privacy of data can be an important aspect in the real-world application of data mining. In privacy-sensitive scenarios, in particular those with distributed data, a failure to guarantee certain privacy-preserving constraints means that data mining can not be applied at all. As an example, consider the case of competing mail order companies. To a large part, these companies make money by knowing their customers better than their competitors do. On the other hand, they lose money due to fraud. Typically, the risk of disclosing sensitive customer information by far outweighs the chances of reducing expenses by a joint fraud detection effort. Only privacy-preserving data mining techniques will allow an analysis of fraud patterns over all companies.

In applications like the above, descriptive techniques like rule mining are very popular, as they have the potential to provide more insight than numerical methods like SVMs or neural networks. Actually, protocols have been proposed that allow secure association rule mining over distributed databases [10]. These, however, rely on the classical support/confidence framework, which has been observed to have effects undesired in some settings [22,3,9]: In particular, there is a danger to come up with huge amounts of rules which are not significant or do not express a correlation.

For this reason, several alternative (non-secure) rule mining approaches have been proposed, that deviate from the classical support/confidence framework. 
These include subgroup discovery [11], contrast set mining [2] and correlated itemset mining [16]. These approaches share many similarities, and are sometimes subsumed under the name supervised descriptive rule discovery [17]. The key differences compared to classical support/confidence association rule mining is (i) the different quality function used to assess the patterns, and (ii) the intention to collect only a small set of $k$ patterns (instead of collecting all patterns satisfying some minimal threshold constraint).

Unfortunately, it is impossible to adapt existing secure association rule mining protocols like [10] to a supervised descriptive rule discovery task like subgroup discovery. The reason lies in the different quality functions. The existing protocols rely on the property that in the support/confidence framework every globally large itemset must be locally large at least at one of the sites. However, an analogous property does not hold for the new quality functions: the globally best rules are not guaranteed to also be among the locally best rules of any site, neither exactly nor approximately [19,24].

In this paper, we present a secure protocol for the task of top- $k$ subgroup discovery. To the best of our knowledge, this is the first approach that tackles any of the above-mentioned supervised descriptive rule discovery tasks. Our approach assumes horizontally partitioned data, that is, all sites use the same set of attributes and the quality of every subgroup depends on all databases. The approach finds patterns in the union of the databases, without disclosing the local databases.

The remainder of this paper is structured as follows: After a brief review of some basic definitions in Section 2, we present our new protocol in Section 3 and prove that it is secure. Next, we describe a prototypical implementation in Section 4 , before we conclude in Section 5 .

\section{Preliminaries}

In this section, we will briefly go over the most important notions from secure multi-party computation and subgroup discovery.

\subsection{Privacy-Preserving Data-Mining and Secure Multi-Party Computation}

Privacy-preserving data mining has emerged to address the situation when the use of data mining techniques is desired, but the data to be mined may not be disclosed or brought together due to privacy considerations. One of the most important lines of research in privacy-preserving data mining is the family of approaches based on secure multi-party computation $[18,7]$. Here, the premise is that the data is distributed over different parties, and the goal is to obtain some result without revealing the (private) data of one party to another. A classical example is Yao' famous millionaires problem [25]: two millionaires want to learn who is richer without revealing the precise amount of their wealth. In the context of data mining, the input would be databases and the result a bunch 
of patterns. We will now review the most important definitions of secure multiparty computation. The following presentation is based on Goldreich [7] (with some simplifications, as we consider a less general scenario).

Multi-Party Computation A multi-party problem, or task, is casted by specifying a mapping from sequences of inputs (one input per party) to sequences of outputs (one output per party). We refer to this mapping as the desired functionality, denoted $f:\left(\{0,1\}^{*}\right)^{S} \rightarrow\left(\{0,1\}^{*}\right)^{S}$, where $S$ denotes the number of parties. That is, $f$ is a mapping of the combined input $\bar{x}=\left(x_{1}, \ldots, x_{S}\right)$ to the combined output $\left(f_{1}(\bar{x}), \ldots, f_{S}(\bar{x})\right)$ : the first party with input $x_{1}$ wishes to obtain $f_{1}(\bar{x})$, the second party with input $x_{2}$ wishes to obtain $f_{2}(\bar{x})$, etc. Clearly, the output of every party can depend on all inputs, $x_{1}, \ldots, x_{S}$.

In this paper, we consider multi-party computation in the semi-honest model, arguably the most commonly used model in privacy-preserving data-mining (e.g. $[13,20,10])$. The semi-honest model assumes that every party follows the protocol properly with the exception that it keeps a record of all intermediate computations and messages. One motivation for the semi-honest model is that parties who want to mine data for their mutual benefit will follow the protocol to get correct results. Another argument is that in a complex software implementation, it might not be easy to deviate from the specified protocol, definitely more difficult than merely recording the registers and messages. Finally, such records may be available anyway through some standard activities of the operating systems, which makes "totally honest" behavior hard to enforce.

A Definition of Secure Computation Intuitively, a protocol $\pi$ securely computes a functionality $f$ if whatever a semi-honest party can obtain after participating in the protocol could be obtained from the input and output available to that party. This is formalized according to the simulation paradigm, which requires that every party's view in a protocol execution can be simulated given only its input and output. As the actual execution may involve messages based on random numbers, the simulator does not need to generate exactly what is seen during the actual execution. The following definition makes this precise:

Definition 1 Let $f=\left(f_{1}, \ldots, f_{S}\right)$ be a deterministic $S$-ary functionality and $\pi$ be a multi-party protocol for computing $f$. For a party $i$, let view ${ }_{i}^{\pi}(\bar{x})$ denote its view, i.e. its input $x_{i}$ and the sequence of messages it has received. We say that $\pi$ securely computes $f$ in the presence of semi-honest adversaries without collusion if there exist probabilistic polynomial-time algorithms $S_{i}, 1 \leq i \leq S$, also called simulators, such that for every party the view is computationally indistinguishable from the simulation:

$$
\left\{\left(S_{i}\left(x_{i}, f_{i}(\bar{x})\right)\right\}_{\bar{x} \in\left(\{0,1\}^{*}\right)^{S}} \equiv^{C}\left\{\operatorname{view}_{i}^{\pi}(\bar{x})\right\}_{\bar{x} \in\left(\{0,1\}^{*}\right)^{S}}\right.
$$

Here, $\equiv^{C}$ denotes computational indistinguishability. Loosely speaking, the simulations are computationally indistinguishable from the views if for every polynomial-time distinguisher $D$ the probability that $D$ distinguishes a view and the 
simulation decreases super-polynomially in the length of the input. The details can be found in [7]. For our purpose, it is sufficient to note that any random number in a view is computationally indistinguishable from another random number drawn from the same probability distribution.

Yao's Generic Circuit Solution Yao has presented a generic solution that allows the secure evaluation of any two-party functionality $[26,14]$. The solution is based on the encryption and secure evaluation of circuits. While in principle this implies that it is possible to securely compute any kind of (two-party) data mining task simply by using a circuit calculating the outcome of the data mining task, this naive approach would result in huge circuits whose inputs would be entire databases - an approach which is not feasible in practice [18]. However, the use of Yao's circuit encryption scheme can be very useful to securely compute some sub-functionality.

\subsection{Subgroup Discovery}

Subgroup discovery is a supervised descriptive rule learning task. In this paper, we only consider binary labeled data, thus a subgroup description can be seen as the antecedent of a rule whose consequence is the positive class.

Formally, let $\mathcal{A}=A_{1}, \ldots, A_{m}$ be a sequence of $m$ sets we refer to as attributes. Beside these attributes, there is a special binary set $\{+,-\}$ called the label. A data record over $\mathcal{A}$ is an $m+1$-tuple $D=\left(a_{1}, \ldots, a_{m}, l\right) \in A_{1} \times \cdots \times A_{m} \times\{+,-\}$. A database $\mathcal{D}$ over $\mathcal{A}$ is a multiset of data records over $\mathcal{A}$. We use the expression $\mathcal{D}^{+}$to denote the sub-multiset of all +-labeled data records in $\mathcal{D}$. Formally, thus $\mathcal{D}^{+}=\left\{\left(a_{1}, \ldots, a_{m}, l\right) \in \mathcal{D} \mid l=+\right\}$. Similarly, $\mathcal{D}^{-}$denotes the sub-multiset of all --labeled data records in $\mathcal{D}$.

The subgroup description language considered here is the language of conjunctions of attribute/value equality constraints. We formalize this as follows: a constraint over $\mathcal{A}$ is an expression $\left(A_{i}=v\right)$ with $i \in\{1, \ldots, m\}$ and $v \in A_{i}$. The language of subgroup descriptions over $\mathcal{A}$, denoted by $\mathcal{L}_{\mathcal{A}}$, is then the power set of constraints over $\mathcal{A}$. In the following, we drop the index $\mathcal{A}$ because it is always clear from the context. Given a subgroup description $s \in \mathcal{L}$, we call the number of constraints it is built of its length, denoted by length $(s)$.

We will now turn to the semantics of subgroup descriptions: a data-record $\left(a_{1}, \ldots, a_{m}, l\right) \in \mathcal{D}$ satisfies a subgroup description $s \in \mathcal{L}$, if for all $\left(A_{i}=v\right) \in s$ it holds that $a_{i}=v$. Then the extension of $s$ in $\mathcal{D}$, denoted by $\mathcal{D}[s]$, is the sub-multiset of $\mathcal{D}$ containing the data records that satisfy $s$.

The "interestingness" of a subgroup description is measured by a quality function, which is a mapping from a database and a subgroup description to the reals. The idea is that high function values indicate interesting patterns. In this paper, we consider the Piatetsky-Shapiro quality function, which is (factor) equivalent to the weighted relative accuracy [12]. This function, which is arguably one of the most common subgroup quality functions, is defined as follows:

$$
q(\mathcal{D}, s)=n(\mathcal{D}, s)\left(p(\mathcal{D}, s)-p_{0}(\mathcal{D})\right) .
$$


Here, $n(\mathcal{D}, s)=|\mathcal{D}[s]|$ denotes the size of the subgroup, $p(\mathcal{D}, s)=\left|\mathcal{D}^{+}[s]\right| /|\mathcal{D}[s]|$ the fraction of records with positive label in the subgroup and $p_{0}(\mathcal{D})=\left|\mathcal{D}^{+}\right| /|\mathcal{D}|$ the fraction of positive records in the overall population. Subgroup discovery is concerned with finding high-quality subgroups, as precised in the next section.

\section{Distributed Secure Subgroup Discovery}

We assume that there are $S \geq 2$ sites (or parties) participating in the computation, each holding a private database $\mathcal{D}_{i}(1 \leq i \leq S)$ built over the same set of attributes. Instead of directly considering the task of top- $k$ subgroup discovery, we first consider a specialization of this problem, namely finding a single subgroup of maximum quality. This task can be seen as the special case where $k=1$. We will describe later (in Section 3.4), how given a solution to this task, we can iteratively collect a set of $k$ subgroups. Beside the subgroup description, we want to learn its quality, because this tells us whether the subgroup describes a significant phenomenon or merely a manifestation of noise in the data. The task we consider is thus the following:

Task 1 Top-1 Subgroup Discovery Given private databases $\mathcal{D}_{1}, \ldots, \mathcal{D}_{S}$ at Sites 1 to $S$ (each built over the same set of attributes) together with a length limit $L$, calculate and distribute a maximum quality subgroup description $s_{\max }$ of length $\leq L$ together with its quality. That is, compute a pair $\left\langle s_{\max }, q_{\max }\right\rangle \in \mathcal{L} \times \mathcal{R}$ such that

$$
\begin{gathered}
q_{\max }=\max _{\{s \in \mathcal{L} \mid \text { length }(s) \leq L\}} q(\mathcal{D}, s), \\
\text { length }\left(s_{\max }\right) \leq L \text { and } q\left(\mathcal{D}, s_{\max }\right)=q_{\max }
\end{gathered}
$$

where the quality function $q$ (defined in Equation 2) is evaluated wrt. the disjoint union of the local databases, $\mathcal{D}=\bigoplus_{i=1}^{S} \mathcal{D}_{i}$.

It turns out that Task 1 is unexpectedly hard: First, as shown by Scholz [19], the globally best rules may perform poor at all local sites, and moreover the local quality of a subgroup can arbitrarily deviate from its global quality (no matter whether a relative or an absolute definition of support is applied). The consequence is that separately analyzing one (or all) local databases is of no help in finding the best global subgroup. This is unlike the situation in the classical support/confidence framework, where every globally large itemset must be locally large at least at one of the sites, a property exploited in protocols like $[4,10]$. The reason for this difference is the different quality function used in subgroup discovery. As a consequence, the distributed association rule mining protocols cannot be adapted to the task of subgroup discovery, and instead a distributed global subgroup mining protocol has been proposed [24] which computes the global quality of every subgroup essentially by polling the local support counts from all participating sites.

In the context of secure computation, we face an additional difficulty: the standard approach of non-secure subgroup discovery algorithms - keeping track 
of the best subgroups observed so far together with their quality during the traversal of the search space $[23,8,24]$ - results in a security leak. The reason is, loosely speaking, that the sequence of increments of the best quality cannot be simulated from the outcome. This sequence, however, reveals a lot of information about the data, as it induces a partial order over the quality of all subgroups visited during the exploration of the search space. For this reason, we compute the maximum-quality subgroup in two steps, by subsequently solving the following two sub-tasks:

- first, we compute the maximum of all subgroup qualities, that is, the value $q_{\max }$. This is done in a way that only the maximum becomes known, but no ordering between subgroup qualities;

- second, we use this quality to securely find a maximum-quality subgroup.

We will discuss these two steps in Sections 3.1 and 3.2, respectively, before we present the overall protocol in Section 3.3 and turn to the task of top- $k$ subgroup discovery in Section 3.4 .

\subsection{Computing the Maximum Quality}

Our solution to the first sub-task works as follows: In a first phase, the sites collectively traverse the space of subgroup descriptions. For every subgroup description, the protocol ensures that Site 1 obtains a random value $r_{i}$, and Site $S$ a second value $\tilde{q}_{i}+r_{i}$, where $\tilde{q}_{i}$ represents the quality of the subgroup (actually, $\tilde{q}_{i}$ is an integer-valued multiple of the subgroup quality, as explained below). The motivation for the distributed storage of $r_{i}$ and $\tilde{q}_{i}+r_{i}$ is that none of the parties must learn the value $\tilde{q}_{i}$. In a second phase, Site 1 and Site $S$ use the garbled qualities $\tilde{q}_{i}+r_{i}$ and the offsets $r_{i}$ to securely calculate the maximum quality. We remark that the first phase is inspired by the garbled quality calculation in [5], while the second phase shares some similarity with the maximum computation in $[13]$.

Computing the Garbled Qualities $\tilde{\boldsymbol{q}}_{\boldsymbol{i}}+\boldsymbol{r}_{\boldsymbol{i}}$. We will now describe these two phases in more detail. We first observe that the Piatetsky-Shapiro quality (Equation 2) can be rewritten as follows $[6]: q(\mathcal{D}, s)=\left|\mathcal{D}^{+}[s]\right|\left(1-p_{0}(\mathcal{D})\right)-$

$\left|\mathcal{D}^{-}[s]\right| p_{0}(\mathcal{D})$. Given that $\mathcal{D}=\bigoplus_{i=1}^{S} \mathcal{D}_{i}$, this means that the quality can be expressed as a sum of local values:

$$
q(\mathcal{D}, s)=\sum_{i=1}^{S}\left(\left|\mathcal{D}_{i}^{+}[s]\right|\left(1-p_{0}(\mathcal{D})\right)-\left|\mathcal{D}_{i}^{-}[s]\right| p_{0}(\mathcal{D})\right)
$$

All that is required to compute the local summands is the value $p_{0}(\mathcal{D})$. Moreover, all summands are multiples of $1 /|\mathcal{D}|$, because $p_{0}$ is a multiple of $1 /|\mathcal{D}|$. Thus, assuming that every site has knowledge of $|\mathcal{D}|$, we can reduce the computation to arithmetics on integers. In fact, the $\tilde{q}_{i}$ mentioned earlier are simply the integers obtained by multiplying the quality of the $i$-th subgroup and $|\mathcal{D}|$. As final observation, we note that the values of the integers $\tilde{q}_{i}$ do not exceed $|\mathcal{D}|^{2}$. 
Based on these observations, we realize the computation of the values $r_{i}$ and $\tilde{q}_{i}+r_{i}$ at Sites 1 resp. $S$ as follows: Site 1 generates a random number $r_{i}$ uniformly distributed in $[0, \ldots, M]$, where $M$ is a some power of 2 constant such that $M>|\mathcal{D}|^{2}$. Site 1 adds it local support, $\left(\left|\mathcal{D}_{1}^{+}\left[s_{i}\right]\right|\left(1-p_{0}\right)-\left|\mathcal{D}_{1}^{-}\left[s_{i}\right]\right| p_{0}\right) \cdot|\mathcal{D}|$ to $r_{i}$, and sends the sum (modulo M) to Site 2. Sites 2 to $(S-1)$ add their local support and send the result to the next site. Finally, Site $S$ obtains the result, which according to Equation 3 corresponds to $\tilde{q}_{i}+r_{i}$.

Obtaining the Maximum Once the first phase of the computation is completed, Site 1 has a list of values $r_{1}, \ldots, r_{R}$, and Site $S$ another list $\tilde{q}_{1}+r_{1}, \ldots, \tilde{q}_{R}+$ $r_{R}$, where $R$ denotes the number of subgroup descriptions visited during the traversal. In the second phase, only two sites are involved. This is an important difference to the first phase, as it means that now we could apply a generic two-party solution like Yao's encrypted circuit scheme [26].

However, due to the potentially very large number of inputs this may result in an in-feasibly large circuit, so we reduce the problem to smaller subproblems: We successively shorten the two lists by iteratively replacing two values by one at both Sites 1 and $S$. Thereby, we take care that while the length of the two lists decrease, together they still allow the reconstruction of $q_{\max }$. This is done by replacing two values $r_{\alpha}, r_{\beta}$ at Site 1 by a new random value $r^{\prime}$, and the two corresponding values $r_{\alpha}+\tilde{q}_{\alpha}, r_{\beta}+\tilde{q}_{\beta}$ at Site $S$ by $r^{\prime}+\max \left(\tilde{q}_{\alpha}, \tilde{q}_{\beta}\right)$. The use of a new random number $r^{\prime}$ is important, as it will allow us to prove that the parties learn nothing new during the replacements ${ }^{1}$. The replacements take place until only one pair of values remains, whose difference reveals the quality $q_{\max }$. Figure 1 illustrates the overall idea.

To put the above into action, all we need is a secure solution for the following functionality: Provided Site 1's input $\left(r_{\alpha}, r_{\beta}, r^{\prime}\right)$ and Site $S$ 's input $\left(r_{\alpha}+\tilde{q}_{\alpha}, r_{\beta}+\tilde{q}_{\beta}\right)$, calculate the combined output $\left(\perp,\left(\max \left(\tilde{q}_{\alpha}, \tilde{q}_{\beta}\right)+r^{\prime}\right) \bmod M\right)$, i.e. Site 1 learns nothing and Site $S$ learns the garbled maximum. Here, all inputs and outputs are integers in $[0, \ldots, M]$.

\footnotetext{
${ }^{1}$ Simply dropping one of the pairs would not be secure, as it would reveal which subgroup has the higher quality; this also explains why we cannot store an encrypted representation of the subgroup description together with its garbled quality
} 
We realize this functionality using an encrypted circuit [26]. A detailed description of the circuit encryption scheme is beyond the scope of this paper (see the excellent presentation in [14] for details). The bottom line is that given some boolean circuit, this scheme allows to generate an encrypted representation of that circuit, together with an encoding table for the boolean input gates. Given this representation, plus cryptographic keys representing a particular set of boolean inputs, it is possible to calculate the (plain) value of the boolean outputs - but no additional information beyond that.

Using this encryption scheme, Site 1 proceeds as follows: First, it generates a boolean circuit that computes the above functionality. The circuit has input wires for the boolean representation of $r_{\alpha}, r_{\beta}, \tilde{q}_{\alpha}+r_{\alpha}, \tilde{q}_{\beta}+r_{\beta}$ and $r^{\prime}$, and the output wires represent $\left(\max \left(\tilde{q}_{\alpha}, \tilde{q}_{\beta}\right)+r^{\prime}\right) \bmod M$. The circuit calculates the output using some inverters and ripple-carry adders, a well-known type of digital circuit. Site 1 generates the encrypted representation of the circuit and sends the circuit (without the encoding table) to Site $S$, together with the keys representing its own input bits. Now all that Site $S$ needs to calculate the output are the keys representing its own input. For this purpose, we make use of an additional ("third") party, $T$ : Site 1 sends the encoding table for the inputs of Site $S$ to Site $T$. Site $S$ sends its (plain) input bits to Site $T$ and obtains the corresponding cryptographic keys. Given this information, Site $S$ can evaluate the encrypted circuit and thus obtain $\left(r^{\prime}+\max \left(\tilde{q}_{\alpha}, \tilde{q}_{\beta}\right)\right) \bmod M$. None of the parties learn anything else than the result. We remark that if $S>2$, the role of Site $T$ can be implemented by one of the sites $2, \ldots,(S-1)$, e.g. Site 2 , after some minor preprocessing. ${ }^{2}$

\subsection{Computing a Maximum Quality Subgroup}

Once the maximum quality $q_{\max }$ is computed, it is straightforward to solve Task 1 and compute a maximum quality subgroup. Basically, all that has to be done is to check, for every subgroup $s_{i}$, whether $q_{i} \geq q_{\max }$, which is equivalent to $\tilde{q}_{i}+r_{i} \geq r_{i}+q_{\max } \cdot|\mathcal{D}|$. The first subgroup satisfying this inequality is returned as outcome. The values $\tilde{q}_{i}+r_{i}$ are known at Site $S$ after execution of Protocol 1, and moreover the values $r_{i}, q_{\max }$ and $|\mathcal{D}|$ are known at Site 1 , so all that is needed is to securely compute the greater-or-equal test. This test, however, is equivalent to Yao' famous millionaires problem [25], and many solutions exist for this task (e.g. [21]).

\subsection{The Protocol}

We now have all ingredients for a secure solution for Task 1. Protocol 1 assumes the length limit $L$ as input, together with local database $\mathcal{D}_{i}, i, 1 \leq i \leq S$.

\footnotetext{
${ }^{2}$ All that is required is that before the circuits are generated, Site 1 and Site $S$ replace every $r_{i}$ by $r_{i}+r_{i}^{\prime}$, where $r_{i}^{\prime}$ is a newly generated random value. This prevents the third party from drawing conclusions by relating the observed inputs from Site $S$ with the intermediate sums observed earlier.
} 
Protocol 1 Maximum Subgroup Quality Computation

INPUT: length limit $L$ and local databases $\mathcal{D}_{1}, \ldots, \mathcal{D}_{S}$

1: Site 1 initiates the secure calculation of $|\mathcal{D}|$ and $\left|\mathcal{D}^{+}\right|$and broadcasts the result

2: Site 1 creates a local iterator iter and a queue $\mathcal{Q}_{1}$, Site $S$ creates a local queue $\mathcal{Q}_{S}$

3: while hasNext(iter) do

4: $\quad$ Site 1 calculates and broadcasts $s_{i}=\operatorname{next}($ iter $)$

5: $\quad$ Site 1 generates a random number $r_{i}$ uniformly in $[0, \ldots, M]$, enqueues $r_{i}$ in $\mathcal{Q}_{1}$, adds its local support $\left(\left|\mathcal{D}_{1}^{+}\left[s_{i}\right]\right|\left(1-p_{0}\right)-\left|\mathcal{D}_{1}^{-}\left[s_{i}\right]\right| p_{0}\right) \cdot|\mathcal{D}|$ to $r_{i}$ and sends the result $(\bmod M)$ to Site 2

6: $\quad$ Sites $2, \ldots, S-1$ add their local support to the intermediate sum and send the result $(\bmod M)$ to the next site

7: $\quad$ Site $S$ adds its local support to the sum and enqueues the result, $\tilde{q}_{i}+r_{i}(\bmod$ $M)$, in $\mathcal{Q}_{S}$

8: end while

9: while $\mathcal{Q}_{1}$ contains more than 1 value do

10: $\quad$ Site 1 dequeues $r_{\alpha}$ and $r_{\beta}$ from $\mathcal{Q}_{1}$, generates a random number $r^{\prime}$ uniformly in $[0, \ldots, M]$ and enqueues $r^{\prime}$ in $\mathcal{Q}_{1}$

11: $\quad$ Site 1 generates and encrypts a circuit that computes $\left.\left(\max \left(\tilde{q}_{\alpha}, \tilde{q}_{\beta}\right)+r^{\prime}\right) \bmod M\right)$ from $r_{\alpha}, r_{\beta}, \tilde{q}_{\alpha}+r_{\alpha}, \tilde{q}_{\beta}+r_{\beta}$ and $r^{\prime}$. It sends the circuit to Site $S$ together with the cryptographic keys corresponding to the input bits for $r_{\alpha}, r_{\beta}$ and $r^{\prime}$.

12: $\quad$ Site 1 sends the encoding table for the remaining inputs to Site $T$

13: $\quad$ Site $S$ dequeues $\left(r_{\alpha}+\tilde{q}_{\alpha}\right)$ and $\left(r_{\beta}+\tilde{q}_{\beta}\right)$ from $\mathcal{Q}_{S}$, asks Site $T$ for the corresponding cryptographic keys, evaluates the encrypted circuit and enqueues the result, $\left(r^{\prime}+\max \left(\tilde{q}_{\alpha}, \tilde{q}_{\beta}\right) \bmod M\right)$, in $\mathcal{Q}_{S}$

\section{4: end while}

15: Sites 1 and $S$ calculate $q_{\max }$ by exchanging the two remaining values

16: for every subgroup descriptions $s_{i}$ do

17: $\quad$ if $\tilde{q}_{i}+r_{i} \geq r_{i}+q_{\max } \cdot|\mathcal{D}|$ then return $\left\langle s_{i}, q_{\max }\right\rangle$

18: end for

OUTPUT: $s_{\max }$ and $q_{\max }$ (same output at all sites)

First, the sites securely calculate $|\mathcal{D}|$ and $\left|\mathcal{D}^{+}\right|$. As $|\mathcal{D}|$ is the sum of the local $\left|\mathcal{D}_{i}\right|$, this reduces to the secure calculation of a sum of local values - a standard task in multi-party computation, for which efficient protocols exist (see e.g. [5]). Same for $\left|\mathcal{D}^{+}\right|$. The values $|\mathcal{D}|$ and $\left|\mathcal{D}^{+}\right|$are distributed to all sites, which will enable them to calculate $p_{0}$ and hence the local values in Equation 3. Next, Site 1 and Site $S$ initialize a local queue, $\mathcal{Q}_{1}$ resp. $\mathcal{Q}_{S}$. These will be used to store the values $r_{1}, \ldots, r_{R}$ (at Site 1) resp. $\tilde{q}_{1}+r_{1}, \ldots, \tilde{q}_{1}+r_{R}$ (at Site $S$ ).

Thereafter, the protocol iterates over all subgroup descriptions (Line 3 to 8 ). This is orchestrated by Site 1, which makes use of an iterator iter to generate all subgroup descriptions satisfying the length limit. The iterator traverses the space of subgroup descriptions in a canonically depth-first, left-to-right order, according to the lexicographic order of the constraints. Using this iterator, Site 1 
generates the next subgroup description $s_{i}$ and informs all sites that $s_{i}$ is the next subgroup description to be considered. Next, the parties collectively calculate $r_{i}$ and $\tilde{q}_{i}+r_{i}$. As discussed earlier, the latter value is computed by iteratively adding the local support and sending the intermediate result to the next site. The values $r_{i}$ resp. $r_{i}+\tilde{q}_{i}$ are separately stored in the queues $\mathcal{Q}_{1}$ and $\mathcal{Q}_{S}$ at Sites 1 and $S$, respectively.

When the second loop starts at Line 9, all candidate subgroups have been considered. The protocol will now determine a pair of values $r_{i^{*}},\left(r_{i^{*}}+\tilde{q}_{i^{*}}\right)$ such that $\tilde{q}_{i^{*}}$ is maximal. This is done by iteratively replacing (a pair of) pairs at Sites 1 and $S$ simultaneously by (a pair of) single values. To this end, in every iteration of Line 11 a new encrypted circuit is generated at Site 1, which is evaluated afterwards by Site $S$, resorting to a third party $T$ (which can optionally be implemented by Site 2 as discussed earlier). The loop ends when only one pair of values remains, which allows the calculation of the quality $q_{\max }$.

Once the figure $q_{\max }$ is calculated, the protocol re-iterates over all subgroups (in Line 16) until a subgroup with maximum quality is met. This subgroup is returned as result together with its quality, and the execution ends.

Protocol 1 is secure, as precised by the following theorem:

Theorem 1. Protocol 1 privately solves Task 1, revealing only $|\mathcal{D}|$ and $\left|\mathcal{D}^{+}\right|$(in the semi-honest model, assuming no collusion).

Proof: We have to specify how every site can simulate its view given the result, the leaked information and its own input. Recall that the simulation does not need to generate the exact same sequence of messages - it suffices that its output is computationally indistinguishable from the view (which involves messages based on random numbers).

The simulator generates execution traces following the algorithmic skeleton of Protocol 1, i.e. by iterating over the subgroup descriptions. This ensures that the simulations have the same overall structure as the views. We will now go over every line of the protocol involving communication and describe how the simulator can generate data computationally indistinguishable from the observed messages. To this end, we will describe how the simulator generates such data in a first paragraph "(S)", before we prove that this data is computationally indistinguishable from the actual messages in a second paragraph "(I)".

Line 1: (S) The protocol computes $|\mathcal{D}|$ and $\left|\mathcal{D}^{+}\right|$using an existing secure-sum subprotocol, e.g. [5]. Goldreich's Composition Theorem [7] says that given secure protocols for sub-tasks, they can be dealt with in a transparent way: all we need to show is that the simulator can predict the outcome of the sub-protocol. Given that $|\mathcal{D}|$ and $\left|\mathcal{D}^{+}\right|$is part of the simulator's input, it can output these very values. (I) The values in the view and in the simulation are computationally indistinguishable because they are identical.

Line 4: (S) The simulator generates the next subgroup using the iterator iter. (I) The subgroup in the view and in the simulation coincide, because the traversal is performed in a canonical way.

Lines 5 to 7: (S) Sites 2 to $S$ generate a random number uniformly in $[0, \ldots, M]$. (I) Given that $r_{i}$ was randomly generated uniformly in $[0, \ldots, M]$, the local sum in the view is also uniformly distributed in $[0, \ldots, M]$. Hence, it is computationally indistinguishable from the random number in the simulation, because two random numbers generated from the same distribution are computationally indistinguishable [7]. 
Lines 11 to 13: The execution of these lines result in the following messages: (i) Site $S$ receives an encrypted circuit representation together with a set of cryptographic keys that allow the evaluation of the circuit; (ii) Site $T$ receives an encoding table, plus the values $\left(r_{\alpha}+\tilde{q}_{\alpha}\right)$ and $\left(r_{\beta}+\tilde{q}_{\beta}\right)$.

(S) For Site $S$, the simulator generates a new encrypted circuit, and uses its representation to simulate the circuit in the view. As simulation of the input $r^{\prime}$, it uses the encryption table to encode the bits of a newly generated random number generated uniformly in $[0, \ldots, M]$. To simulate the other inputs, it uses the encryption table to encode some arbitrary boolean values. For Site $T$, the simulator generates an encrypted circuit and uses the encoding table as simulation of the table in the view. Moreover, it generates two random numbers as simulation of the two inputs from Site $S$.

(I) For Site $S$, recall that all can be extracted from an encrypted circuit, given a particular set of input keys, is the outcome [14]. The outcome of the circuit in the view is a random number uniformly distributed in the domain $[0, \ldots, M]$, which is independent from all values observed so far (recall that Site 1 generates a new random offset in every iteration). The same is true for the circuit in the simulation, thus the view and the simulation are computationally indistinguishable. For Site T, the encoding table in the view is computationally indistinguishable from that in the simulation because both essentially consist of a set of cryptographic keys generates by the same cryptographic key generation mechanism. The inputs from site $S$ in the view are computationally indistinguishable from the random numbers in the simulation because both are uniformly distributed in $[0, \ldots, M]$, and are independent from all values observed so far.

Line 15: (S) The simulator generates $q_{\max }$, which it is part of its input. (I) obvious.

Line: 17: (S) Again, due to the composition theorem the simulator only has to generate the result of the test (plus, optionally, $s_{\max }$ ). This is straightforward, given that $s_{\max }$ is part of the input. (I) obvious.

\subsection{Top- $k$ Subgroup Discovery and the Weighted Covering Scheme}

It is straightforward to extend our solution to collect a set of subgroups. The simplest way is to iteratively collect the $k$ highest-quality subgroups one after another, thereby solving the top- $k$ subgroup discovery task. This only requires a minor modification of the iterator, ensuring that all subgroups collected so far will be ignored during subsequent search space traversals.

A more sophisticated approach would be to use the weighted covering scheme [12]. Here again, the idea is to iteratively search for the maximum subgroup, however using a definition of quality that accounts for record weights. After every iteration, the weights of the records covered by the subgroup collected so far is decreased by multiplication with some rational number. This results in a definition of quality which is equivalent to the following:

$$
q^{w}(\mathcal{D}, s)=\sum_{i=1}^{S}\left(\left|\mathcal{D}_{i}^{+}[s]\right|^{w}\left(1-p_{0}(\mathcal{D})^{w}\right)-\left|\mathcal{D}_{i}^{-}[s]\right|^{w} p_{0}(\mathcal{D})^{w}\right)
$$

Here, $|\mathcal{D}|^{w}$ denotes the sum of the weights of the records in $\mathcal{D}$, and similarly $p_{0}(\mathcal{D})^{w}=\left|\mathcal{D}^{+}\right|^{w} /|\mathcal{D}|^{w}$. All that needs to be done to implement the weighted 
covering scheme is thus to make use of this quality definition instead of that in Equation 3. Given that Equation 4 is a sum of local values, and that these values are rational numbers which can be computed locally given the set of subgroups collected in the previous iterations, the adaptation is thus straightforward.

\section{Prototypical Implementation}

Our prototype was implemented in Java. For the encryption of the circuits, we used the AES cipher with 128 bit keys implemented in the lightweight API of the crypto-library Bouncycastle (http://www.bouncycastle.org). To compute secure sums, we used the secure sum protocol described in [5]. For secure comparisons, we used the efficient solution for Yao' millionaires problem described in [21]. The quality calculation is realized without sophisticated data structures like FpTrees, as the prototype is merely intended as a proof-of-concept.

We have evaluated the performance of our implementation on different datasets from the well-known UCI repository [1]. All datasets where randomly split into three parts, which were used as local datasets. The experiments were performed on three Core 2 Duo E8400 PCs with 2GB RAM, connected by an Ethernet LAN. The length limit, $L$, was set to 3 . Figure 2 visualizes the result. Beside the overall runtime ("total"), it shows the proportions of the runtime spent (i) for the distributed calculation of the garbled subgroup qualities ("qual."), and (ii) for the evaluation of the encrypted circuits ("circ."). These two components correspond to the first resp. second while loop of Protocol 1. The figure shows that the most costly part is the encryption and evaluation of the circuits.

Compared with state-of-the-art non-secure subgroup discovery algorithms $[12,8]$, the computation is (extremely) slow. Nevertheless, the experiments show that our approach is applicable in practice. The runtime is sufficient to process data sets of realistic size in a few hours, which is quite often sufficiently fast for practical use. In scenarios where a failure to guarantee privacy means that data mining can not be applied at all, the users may very well be willing to invest this time if that allows to find valuable patterns which could not be obtained otherwise.



Fig. 2. Runtime of the prototype

\section{Conclusions}

In many pattern mining settings, the data to be analyzed is sensitive, which makes the use of privacy-preserving techniques mandatory. Although approaches 
exist to securely find association rules in distributed data, these cannot be adapted to supervised descriptive mining tasks like subgroup discovery. The source for the difficulties are precisely the features that distinguish subgroup discovery from classical association rule mining: (i) the different quality function, and (ii) the aim to collect only $k$ patterns.

In this paper, we have presented a new secure protocol that allows secure subgroups discovery on horizontally partitioned data. While the basic protocol only solves the top-1 subgroup discovery task, it can be iterated to collect a set of $k$ subgroups. We have analyzed the properties of our protocol and have shown that it leaks only little information, namely the size of the database and the share of positive records. Finally, we have reported on a prototypical implementation and experiments which demonstrate the feasibility of the approach.

In the experiments it has become clear that the improvements in security and privacy come at the price of a high runtime. While the worst-case complexity of our algorithm is the same as for a non-secure solution, i.e. exponential in the number of attributes, in practice it is much slower than the latter. One reason is of course the slowdown caused by communication and encryption overhead. Another reason is that approaches of speeding up subgroup discovery, such as optimistic estimate pruning [8] or local counting pruning [24] were not considered here. The reason is that they need to exchange additional information between local parties, which make them problematic in a secure computation setting. It would be worthwhile to investigate the effective severity of such information leaks. Clearly, knowledge about optimistic estimates tells something about the private data, but it is not really clear how much. In particular, does it allow to reconstruct (part of) the data? This question is closely related to the so-called task of inverse frequent set mining [15]. We leave the investigation of these issues to future work.

Another interesting question is whether the protocols presented in this paper can be adapted to other quality functions used in supervised descriptive rule discovery. Finally, it would be desirable to extend the security guarantees to colluding parties. One standard approach in the cryptographic community to deal with collusion issues is to divide the information into different parts, and

to use different routes for the different calculations (e.g. [10]). Whether such an approach is applicable is left to future work.

\section{References}

1. A. Asuncion and D. Newman. UCI machine learning repository, 2007.

2. S. D. Bay and M. J. Pazzani. Detecting group differences: Mining contrast sets. Data Min. Knowl. Discov., 5(3):213-246, 2001.

3. S. Brin, R. Motwani, and C. Silverstein. Beyond market baskets: Generalizing association rules to correlations. In J. Peckham, editor, SIGMOD Conference, pages 265-276. ACM Press, 1997.

4. D. Cheung, J. Han, V. Ng, A. Fu, and Y. Fu. A fast distributed algorithm for mining association rules. Parallel and Distributed Information Systems, International Conference, 0:0031, 1996. 
5. C. Clifton, M. Kantarcioglu, J. Vaidya, X. Lin, and M. Y. Zhu. Tools for privacy preserving distributed data mining. SIGKDD Explor. Newsl., 4(2), 2002.

6. J. Fürnkranz and P. A. Flach. Roc 'n' rule learning-towards a better understanding of covering algorithms. Machine Learning, 58(1):39-77, 2005.

7. O. Goldreich. The Foundations of Cryptography, volume 2, chapter General Cryptographic Protocols. Cambridge University Press, 2004

8. H. Grosskreutz, S. Rüping, and S. Wrobel. Tight optimistic estimates for fast subgroup discovery. In ECML/PKDD (1). Springer, 2008.

9. W. Hämäläinen and M. Nykänen. Efficient discovery of statistically significant association rules. In ICDM, pages 203-212. IEEE Computer Society, 2008.

10. M. Kantarcioglu and C. Clifton. Privacy-preserving distributed mining of association rules on horizontally partitioned data. IEEE Transactions on Knowledge and Data Engineering, 16(9):1026-1037, 2004.

11. W. Klösgen. Explora: A multipattern and multistrategy discovery assistant. In Advances in Knowledge Discovery and Data Mining. 1996.

12. N. Lavrac, B. Kavsek, P. Flach, and L. Todorovski. Subgroup discovery with cn2-sd. Journal of Machine Learning Research, 5(Feb):153-188, February 2004.

13. Y. Lindell and B. Pinkas. Privacy preserving data mining. In CRYPTO, volume 1880 of Lecture Notes in Computer Science. Springer, 2000.

14. Y. Lindell and B. Pinkas. A proof of yao's protocol for secure two-party computation. Technical report, 2004.

15. T. Mielikäinen. On inverse frequent set mining. In Workshop on Privacy Preserving Data Mining, 2003.

16. S. Nijssen, T. Guns, and L. D. Raedt. Correlated itemset mining in roc space: a constraint programming approach. In KDD, pages 647-656, 2009.

17. P. K. Novak, N. Lavrač, and G. I. Webb. Supervised descriptive rule discovery: A unifying survey of contrast set, emerging pattern and subgroup mining. Journal of Machine Learning Research, 10, 2009.

18. B. Pinkas. Cryptographic techniques for privacy-preserving data mining. SIGKDD Explor. Newsl., 4(2):12-19, 2002.

19. M. Scholz. On the tractability of rule discovery from distributed data. In ICDM, pages 761-764. IEEE Computer Society, 2005.

20. M. Shaneck, Y. Kim, and V. Kumar. Privacy preserving nearest neighbor search. In ICDM Workshops, pages 541-545, 2006.

21. L. Shundong, D. Yiqi, W. Daoshun, and L. Ping. Symmetric encryption solutions to millionaire's problem and its extension. In 1st International Conference on Digital Information Management, 2006.

22. G. I. Webb. Discovering significant rules. In KDD '06: Proceedings of the 12th ACM SIGKDD international conference on Knowledge discovery and data mining, pages 434-443, New York, NY, USA, 2006. ACM.

23. S. Wrobel. An algorithm for multi-relational discovery of subgroups. In J. Komorowski and J. Zytkow, editors, Proc. First European Symposion on Principles of Data Mining and Knowledge Discovery (PKDD-97), pages 78-87. Springer, 1997.

24. M. Wurst and M. Scholz. Distributed subgroup mining. In PKDD, 2006.

25. A. C.-C. Yao. Protocols for secure computations (extended abstract). In FOCS. IEEE, 1982.

26. A. C.-C. Yao. How to generate and exchange secrets. In Foundations of Computer Science, 1985., 27th Annual Symposium on, pages 162-167, Oct. 1986. 